\title{
THE
}

\section{DUBLIN JOURNAL OF MEDICAL SCIENCE}

\section{THE OFFICIAL ORGAN OF THE ROYAL ACADEMY OF MEDICINE IN IRELAND}

FOURTH SERIES. No. 21. NOVEMBER, 1921

\section{Original Communications.}

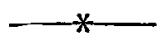

I.

\section{MENSTRUATION AND OVULATION.}

\author{
By Professor Dr. Oskar Frankl, Vienna.
}

W R. CHAIRMAN, Members of the Royal Academy 11 of Medicine, Ladies and Gentlemen,

I wish to thank you most heartily for your cordial invitation to deliver three lectures before you. I begin my address with two different feelings. The first is a feeling of hesitancy, whether I will succeed to arouse your interest and whether I will be able to tell you anything that has not been familiar to you this long time. The second feeling is that of pride and pleasure that you have invited me, a son of the poorest country in the world-namely, Austria. I shall be able to show you that in spite of our poverty we are not at a stand-still in the field of science, but that we intend to continue our researches until the foreign countries will again grant us the friendship and appreciation which we enjoyed in such a full measure before the war. It will be my ambition not only to serve science, but it will also be my great endeavour to regain old and to win new friends for our poor fatherland. It is for this that $I$ have willingly absented myself from my work in Vienna. I consider it a very great honour to be allowed to speak before this old and honourable Royal Academy of Medicine, and I shall 
make the attempt to the best of my ability to bring before you the three papers which I have prepared for this occasion.

The inter-relationship between menstruation and ovulation is of the highest importance not only to the gynæcologist, but to every practitioner who is concerned with the treatment of diseases of women. Menstruation plays an important rôle in various ways in their physiological and pathological economy. It is therefore of fundamental importance that we should have a clear idea of the phenomena of the expulsion of the ovum and of menstrual bleeding.

But the question is of much wider interest. For we know to-day that menstruation and ovulation are in mutual relationship to one another, and also that these two functions are closely associated with the functioning of other organs, especially the various ductless glands. Therefore if we wish to investigate thoroughly the question of the processes of menstruation and ovulation, it is necessary to study the most important relations between the genital organs and the glands of internal secretion.

The functions of the female genital organs can continue regularly and satisfactorily only if the balance between the different ductless glands (including certain parts of the ovary, which we know produce hormones) is maintained. The disappearance or the hyperfunction of a gland immediately produces a disturbance in the physiological balance of the female economy, which has been called " dyshormonia." This effect might be compared to the constituent parts of an orchestra which are not tuned properly (Seitz). The instruments are the ductless glands and the harmonious melody produced the general well-being of the body. Faulty playing on the part of one performer produces a discordant effect on the tout ensemble- the whole melody is adversely affected. We have here in short a dyshormonia.

In this connection the hypophysis, in my opinion, plays a most important part. We know that the anterior lobe of the hypophysis is an endocrine gland and secretes a hormone whose chemical composition has as yet not been very well defined. The posterior lobe consists for the most part of nerve tissue, but is surrounded also by a thin layer of epithelial tissue, which also secretes a hormone. The 
chemical constituents of this latter secretion are a little better known; it is a derivative of Imidazole.

The investigations of Cushing and Aschner have shown that extirpation of the anterior lobe in young animals causes a stagnation of the general development of the body, and particularly of the genital organs, whilst animals fed on a diet containing anterior lobes substance develop and grow rapidly, and their genital organs are quickly and intensively developed. On the other hand we know that during pregnancy (Erdheim and Stumme) and after castration (Tandler and Gross) the hypophysis tends to somewhat hypertrophy.

During pregnancy the essential cells show an enlargement, but after castration the increase is due to the eosinophil cells.

In cases of tumors which destroy the anterior lobe, there appears a condition known as dystrophia adiposo-genitalis, that is a development of a super-abundance of adipose tissue throughout the body with involution of the genitalia and of the secondary sexual characteristics. Where there is an excessive growth of the anterior lobe during youth acromegaly is produced, that is gigantism, which is accompanied by genital hypoplasia. Geller found that after treatment of the hypophysis by $x$-rays the growth and development of the body ceaser, and likewise that of the genital organs. On the other hand, an extract of the posterior lobe of tho pituitary gland causes an increase of blood pressure and contractions of the uterus, very similar to the effects produced by ergotin (Fröhlich and von Frankl). Hofbauer was the first man to show the stimulating effect of pituitrin on the contractions of the uterus and who recommended it for general use in the midwifery.

The successful treatment of amenorhoea by means of pituitrin, first originated by Fromme, Kosminsky, Hofstätter, also points to the close relationship between the hypophysis and the genital organs.

It is therefore reasonable to conclude that a diminution of the genital function may be caused by a dysfunction of the hypophysis; but we know also that the other ductless glands as well as certain parts of the brain, and particularly the region of the third ventricle, may play a rôle in this con- 
nection. In cases of a defective condition of the pituitary gland there is apparently an absence of the hormone, which stimulates the ovary to function.

So we may conclude that for the normal process of menstruation, which on its part is dependent upon a proper function of the ovary, an intact hypophysis is essential.

You will no doubt be surprised to learn that I speak next of the pineal gland as being second in importance of the ductless glands. As Marburg and Biedl have shown, the pineal gland is most active during early infancy, and perhaps also during intra-uterine life. The hyperfunction of this gland at a later period is of great importance for the individual. We know that the development of adipose tissue in the fetus and child is in close relationship with the proper function of this gland. And we also know from experiments and clinical experiences that the pineal gland restrains the growth of the sexual organs, and that the absence of its full function provokes a premature sexual activity. It is also established that with tumors of the pineal gland premature genital development takes place, and that the absence of this gland brings about a genital development which should normally occur much later. Hofstätter believes that he has successfully treated a pathological increase of sexual desire of an imbecile child by means of epiglandol, that is, a watery extract of the pineal gland.

We know that diseases of the pineal gland may produce an abnormal deposition of fat, and that this gland also influences the vasomotor system of the skin-vessels; both these effects play an important rôle in the sexual life of a wornan. Just consider the production of adipose tissue and the vasomotor disturbances which are associated with the onset of the menopause, and you will grant that there are close connections between genital organs and the pineal gland, which, although as yet are not perfectly understood, exist we know without doubt.

Of profound interest are the relations of the thyroid to genital functions. It is known that this gland shows a swelling during puberty, pregnancy and at each menstrual period. At this point may be mentioned Zuckermann's observation, that with congenital aplasia of the thyroid an 
enlargement of the hypophysis takes place. I myself have studied the relation of the thyroid to the genital functions especially in cases of exophthalmic goitre. In these cases I proved that the thyrotoxin frequently produces even in the early stages of the disease an ovarian lesion and amenorrhœa.

In order to have a clear understanding of the inter-relationship between the ovary and the thyroid, it is necessary to study their respective relationship upon the sympathetic system. And there we discover the following facts. The ovary and the chromaffinic system are antagonists. The absence of the ovary protects the chromaffinic system and consequently the sympathetic system. On the other hand, the thyroid furthers the chromaffinic system and increases the irritability of the sympathetic nerves. Therefore the thyroid and the chromaffinic system are synergists.

The ovary decreases, the thyroid increases the blood pressure. During the climacteric and after castration we find increased blood pressure, and accelerated pulse rate. Therefore we observe that the ovary and the thyroid in their relation to the sympathetic system have diagonally opposite effect. In climacteric women the sweating, the trembling and the heart-beat certainly depend upon the thyroid. The increase of adipose tissue is probably due to the hypophysis, the flushings to the adrenals, as far as the sympathetic nerves of the head are influenced by the adrenals. It is to be remarked that the removal of the thyroid in young animals provokes disappearance of the follicles and a hypoplastic condition of the ovary. Lanz found that after extirpation of the thyroid in goats sterility and atrophy of the mammæ occurs. Also in myxodema we find regularly involution of the genital organs.

We are not astonished to find that the adrenals work in close connection with the genital system, since we are informed through Takamine (1901) about the qualities of the adrenaline. Nowak found after extirpation of both adrenals in rats either a hypoplasia or an atrophy of the genital organs, as well as after artificially produced tumors of the adrenals. On the other hand, we find through the experiments of Schenk a hypertrophy of the adrenals after castration. However, this does not indicate an increase of the 
produetion of adrenaline, but, on the contrary, the production of adrenaline is diminished in the hypertrophy of the cortex of the adrenals, since it is a secretion of the marrow

The inter-relationship of the chromatinic system either to the ovary or to the thyroid have already been discussed. Towards the end of pregnancy the adrenaline production shows an increase, and Kehrer is of the opinion that it plays an important part in the onset of labour.

In this connection I wish briefly to speak about the thymus. The maturing of the ovary brings about it the physiological involution of the thymus. On the other hand, the status thymico-lymphaticus always is accompanied by hypoplasia of the genital organs.

Therefore it is obvious that the function of the genital organs through many channels is connected with the ductless glands. So now we will be able to fully comprehend the rôle of the ovary as a regulating central organ for the onset of the menstrual period.

Menstruation is influenced by a rhythmic increasing and decreasing influx of blood into the uterus. The cyclic change of the blood supply becomes regulated through the hormone, which is elaborated in the ovary and absorbed in the bloodstream. This hormone is produced only then, when the ovary is maintained in proper functionating condition through checking and spurring on of the other ductless glands. All other ductless glands act in a protecting or checking manner, but at all events they act as a determining factor upon the proper function of the ovary.

Now we are prepared to study the time and casual relations between the different phases of the ovarian activity and the uterine function. Which portions of the ovary are to be considered as hormone-producing tissues? Unduubtedly the ovum cell is of highest significance. If the ovum dies, then the corpus luteum is involved; if the ovum becomes imbedded, it influences a persistence of the corpus luteum. Therefore we can conclude that the involution of the corpus luteum marks the death of the ovum. Since the beginning involution of the corpus luteum is synchronous with menstruation, we can also conclude that menstruation marks the already established death of the ovum. 
If the ovum becomes imbedded in the mucous membrane, then the corpus luteum persists and menstruation does not take place. Bleedings during pregnancy are without exception pathological, and originate usually from erosions or polypi.

Also the follicular epithelium during all stages of maturing of the follicle, especially the corpus luteum, derived from it, is of inner secretory significance. The older investigators, Pflüger, Leopold and Ravano, were still of the opinion that ovulation and menstruation are coincident. It is to the merit of Fraenkel, in Breslau, to have found that the follicle ruptures between two menstruations. Based upon exact microscopical examinations, performed by Meyer, Ruge, Schroeder, we know to-day that the rupture of the follicle occurs between the 14 th and 16th day after the last menstruation. There exists an ovarian and a uterine cycle, and between them exists the closest relation-one is dependent upon the other. After the follicle is ruptured, the corpus luteum is formed. According to the various stages of development of the corpus luteum we distinguish a stage of proliferation, vascularisation, matured stage and different stages of involution, whose end effect is the so-called corpus albicans or the corpus fibrosum.

The mucosa of the uterus shows a very different construction depending upon the stage within the cycle from which the specimen was taken. Directly after menstruation the mucosa is thinned out from one half to one millimeter, is anæmic, and the glands are contracted and without secretion. We call this a postmenstrual mucosa. Gradually, during the intermenstrual period, the mucous membrane becomes thickened, and correspondingly increased in vascularity, the glands become distended, more tortuous, with more mucous content. Toward the end of the interval, in the premenstruum, the mucosa has become very thick, to the extent of 10 or 15 millimeters. The mucosa has become more vascular, the vessels are widely dilated, so that the connective tissue has an odematous appearance, a most remarkable fact, because we meet for the first time a physiological odema, while we are accustomed to regard œdema as something pathological. The glands are at 
maximum dilatation, tortuous, and full of secretion. Here we must note that only the middle portion of the glands secrete, while the neck and the fundus of the glands remain contracted, inactive. The stroma between the neck of the glands has undergone a change, which consists in a marked swelling of the connective tissue cells, and reminds one to an extent of decidual cells. The menstruating mucosa shows us during the first stage bleedings into the tissue and gradual bleeding into the uterine cavity, and with it a sudden collapse of the whole mucous membrane. Blood pours out of the vessels, mucous out of the glands, œdema fluid out of the stroma, and so it follows that the mucous membrane in a few hours becomes thinned out from a 10 or 15 millimeters diameter to that of half a millimeter. In this way the entire surface may be thrown off, and only the fundus of the glands remains, or there is only a collapse of mucous membrane without any destruction.

The rupture of the follicle occurs between the 13th and 17th day. While the corpus luteum is developing, the secretion of the glands gradually in: creases, the mucosa becomes thicker and prepares itself for the reception of an ovum. If the corpus luteum is at the height of its development, the mucosa is premenstrual. Now the ovum dies. Its long-distant, stimulating effect upon the corpus luteum disappears, the corpus Juteum shows a fatty degeneration. Now menstruation is established. The corpus luteum is transformed into a fibrous body, which soon disappears. At the same time another follicle has developed. As long as the corpus luteum is at the height of its development, it does not allow another follicle to be fully formed. The corpus luteum is an autocrat, and does not tolerate another master while it is itself the master of the situation. It watches over all functions, which must be performed. When the corpus luteum is degenerated, then another follicle may ripen, and the play is repeated.

Thus we can say, and clinical and experimental facts permit of no doubt, that the corpus luteum prepares the mucaus membrane for the reception of an ovun. The corpus luteum operates upon the mucous membrane in a 
constructive sense by its hyperæmia-producing stimulant; this was experimentally proved. But the menstrual bleeding is provoked by the involution of the corpus luteum. Halban and Köhler have established through their clinical observations, that after extirpation of the fully developed corpus lutem, a few days later menstruation sets in. In the same sense are to be taken the experiences of Seitz and Wintz. After Roentgen-castration by one exposure, which was applied at the beginning of the interval, an immediate cessation of menstruation was abserved. However, if the woman was near the end of the interval, the menstruation recurred once before total cessation. The death of the corpus luteum, therefore, produces the menstrual bleeding.

How can we explain this apparent contradiction, that the same hormone produces hyperæmia of the mucous membrane, and its absence provokes the menstrual bleeding? In this case it is necessary to investigate more extensively, and to clear up, in what manner the menstrual bleeding is really brought about. I have pointed out to you that during the premenstrual stage the glands of the mucous membrane are distended with secretion in their middle portion. This mucus contains, as I have demonstrated with Halban and Aschner several years ago through chemical investigations, an abundant tryptic ferment. As long as the corpus luteum gives off its hormone, the mucosa of the uterus is hyperemic, and through the turgor the efferent ducts of the glands are more or less closed. When the corpus luteum begins to involute, the hyperæmia suddenly subsides, the turgor of the tissue becomes diminished, the efferent ducts of the glands are opened, and the mucus escapes. This trypticmucus brings about a digestion of the mucosa, either more superficially or by penetrating more deeply, and casting off of the surface.

The apparent absence of coagulability of menstrual blood, while the blood in the general circulation has retained its normal coagulability, is explained by the fact that the menstrual blood is mixed with tryptic secretion of the uterine glands. This trypsin dissolves fibrin, and so the coagulation of the menstrual blood is prevented. 
Thus it is comprehensibls that the corpus luteum produces a gradually increased hyperæmia, gradually increased thickening and secretion in the mucous membrane. The bleeding appears when the corpus luteum involutes as a consequence of the death of the ovum.

Now, we must shortly consider the pathological aspects of menstruation, which are for you as practitioners of an especial interest. Occasionally it is possible to overcome an amenorrhoea by giving ovarian extract. This can be understood. For, according to the experiments of Aschner, one can produce a hyperæmia of the uterus by ovarian extracts. When the hypophysial impulse is absent it may be possible to re-establish the failing menstruation by pituitrin. And, indeed, we are able in some cases of amenorrhœa, by injections of pituitrin to start the menstrual flow. A very successful means for stimulating the ovarian action we have in the so-called " irritating radiation," this is the application of small doses of $x$-rays to the ovaries. They work in a stimulating manner upon the action of the ovaries, and thus we frequently are enabled to overcome an amenorrhcea. During the late war we so frequently had the opportunity in Vienna of observing amenorrhœa, and could not infrequently prove to our satisfaction the efficiency of the " irritating radiation."

A few words more about meno- and metrorrhagia. I exclude here entirely the myoma bleedings, as these are based upon conditions. altogether different from the true metropathic bleedings. It is probable that through inflammatory processes, and through congestion, a chronic and a permanent hyperæmia of the ovary is induced. This leads to a premature and sudden ripening of the follicles, in which none come to a full development or complete rupture. Therefore, the corpus luteum is lacling. The lining epithelium of the numerous follicles, which have undergone cystic degeneration, produces a chronic hyperæmia of the endometrium, which is followed by hyperplasia, sometimes by polyposis of the mucous membrane. The intensive chronic hyperæmia is sufficient in itself to produce bleedings. The ovary is invaded with many small follicle cysts. The uterus shows an inclination towards meno- and metrorrhagia, 
but without any local inflammatory condition, the condition being exclusively based upon hormone stimulants, which are due to the hypophysis, or to hyperfunction of the ovary.

Since we are unable up to the present time to isolate and subject the hypophysis to treatment, we must resort to $x$-ray treatment and the vaginal extirpation of the uterus in such cases. To what extent abnormal function of the adrenals or the pineal gland play a role in these cases, remains in the field of future investigations. We have here an extensive field of research ahead of us. Just as we are able at the present time to test the function of the liver or the kidney, we must study functional tests for all the ductless glands in future. Only when we have reached this goal, will we be in a position along with other adrantages to influence a typical bleeding without the heroic methods which we employ, such as $x$-ray or extirpation of the uterus.

You have noticed that in this whole exposition the term " interstitial gland" in the sense of "puberty-gland," as Steinach calls it, was not employed. I consider that an interstitial ovarian gland in a sexually matured woman can not be demonstrated histologically, and that up to the present we have no proof either for its existence or for its function. Therefore, I can absolutely not agree with Steinach as to his rejuvenating experiments.

With this I have finished my arguments. I am cognisant of the fact that I have left many loop-holes open. My purpose was to stimulate you to further research work along these lines, and I shall be satisfied if $I$ have succeeded in arousing your interest in this very important field of medical investigation.

II.

\section{EARLY DIAGNOSIS OF CARCINOMA OF THE UTERUS.}

I have taken the early diagnosis of carcinoma of the uterus as the object of my discussion for to-day, because I am of the opinion that this subject is not only of the greatest importance to the pathologist, but also for the general practitioner If it is true that the early diagnosis 